\title{
Targeted modification of regulatory genes associated with barley grain color formation
}

Egorova A. ${ }^{1,2 *}$, Strygina K. ${ }^{4}$, Shoeva O. ${ }^{1}$, Hertig C. ${ }^{3}$, Gerasimova S. ${ }^{1,2}$, Koeppel I. ${ }^{3}$, Hiekel S. ${ }^{3}$, Vikhorev A. ${ }^{1,2}$, Kumlehn J. ${ }^{3}$, Khlestkina E. ${ }^{4}$

${ }^{1}$ Institute of Cytology and Genetics, SB RAS, Novosibirsk, Russia

${ }^{2}$ Novisibirsk State University, Novosibirsk, Russia

${ }^{3}$ Leibniz Institute of Plant Genetics and Crop Plant Research, Gatersleben, Germany

${ }^{4}$ N.I. Vavilov All-Russian Institute of Plant Genetic Resources, St. Petersburg, Russia

*email: egorova@bionet.nsc.ru

Blue and purple colors of barley (Hordeum vulgare L.) grain are caused by anthocyanin accumulation in aleurone and pericarp respectively. Anthocyanins are known for their human health benefits. It is thus desirable to breed and grow barley with colored grains. In our previous experiments, the bHLH transcription factors MYC2 and ANT2 have been shown to be implicated in the control of barley grain pigmentation. In the present investigation, the MYC2 and ANT2 genes of barley were subjected to Cas9/gRNAmediated site-directed mutagenesis aiming to further elucidate the roles of these genes in grain color formation. It was previously shown that the loss-of-function of $H v M Y C 2$ is associated with non-colored compared to blue-grained barley. To provide compelling evidence of MYC2 function in regard to blue aleurone formation, an attempt was made to restore the reading frame of the mutated allele in non-colored barley by deleting the spontaneously inserted nucleotide. To this end, cas $9 /$ gRNA plasmids were constructed for two target sites in the HvMYC2 gene. Barley cultivar 'Golden Promise' with uncolored grains was used as experimental line for the intended modification. After Agrobacterium-mediated transformation, 49 primary mutant plants were obtained, out of which 19 featured the desired loss of a single nucleotide. To confirm the role of ANT2 in purple pericarp formation, a targeted knockout of the respective gene was required in purple-grained barley. To generate experimental lines amenable to genetic transformation, the purple PLP accession was crossed with 'Golden Promise', which was followed by the use of resulting hybrids for the knockout approach. A cas $9 / \mathrm{gRNA}$ construct was generated, containing three gRNAs for different target sites in the HvANT2 gene. Agrobacterium-mediated transformation resulted in 67 primary mutant plants. The phenotypic analysis of the generated mutants is in progress.

Acknowledgements: The work is supported by the RSF (21-66-00012). 